In the Nouveau Bulletin des Sciences, par la Société Philomathique de Paris, tome ii., Paris, I810, occurs this passage:-

"Mathématiques.-Sur les Équations différentielles des Courbes du Second Degré, par M. Monge. L'équation générale des courbes du second degré étant

$$
A y^{2}+2 B x y+C x^{2}+2 D y+E x+1=0,
$$

dans laquelle $A, B, C, D, E$ sont des constantes, M. Monge donne l'équation différentielle débarrassée de toutes ces constantes, et il parvient à l'équation suivante, du cinquième ordre, (A)

$$
9 q^{2} t-45 q q^{r s}+40 r^{3}=0,
$$

les quantités $r, s, t$, étant définies par les équations suivantes:

$$
\frac{d y}{d x}=p, \frac{d p}{d x}=q, \frac{d q}{d x}=r, \frac{d r}{d x}=s, \frac{d s}{d x}=t .
$$

"Il faut ensnite voir l'usage de l'équation (A), pour trouver l'intégrale d'une équation d'un ordre inférieur qui satisfait à cette équation (A); ainsi étant donnée l'équation différentielle $\left(\mathrm{I}+p^{2}\right) r=3 p q^{2}$, il parvient à l'intégrale $(x-a)^{2}+(y-b)^{2}=c^{2}$, qui est l'équation d'un cercle.

"La même méthode pourroit s'appliquer aux équations des tourbes d'un degré supérieur au second."

A note is added to the effect that "Cet article est extrait de la Correspondance de l'Ecole impériale Polytechnique, rédigée par M. Hachette: Ir cahier du 2e volume, 18 1o." The press mark of this work at the British Muse um is PP. I 543.

Trusting that this is the reference you are in search of, and that the long delay in the discovery of it may be excused when the difficulty of identifying a particular passage (known perhaps only in its full extent to those whose chief work is concerned with such matters) is considered.

$I$ remain, Sir, faithfully yours,

Prof. J. J. Sylvester, \&c., \&c.

$$
\text { H. FISHER }
$$

New College, Oxford, April 19

\section{J. J. SYlvester}

On the Velocity of Light as Determined by Foucault's Revolving Mirror

IT has been shown by Lord Rayleigh and others that the velocity $(U)$ with which a group of waves is propagated in any medium may be calculated by the formula-

$$
U=V\left(\mathrm{x}-\frac{d \log V}{d \log \lambda}\right)
$$

where $V$ is the wave-velocity, and $\lambda$ the wave-length. It has also been observed by Lord Rayleigh that the fronts of the waves reflected by the revolving mirror in Fo:icault's experiment are inclined one to another, and in consequence must rotate with an angular velocity-

$$
\frac{d V}{d \lambda} \alpha
$$

where $\alpha$ is the angle between two successive wave-planes of similar phase. When $d V / d \lambda$ is positive (the usual case), the direction of rotation is such that the following wave-plane rotates towards the position of the preceding (see NATURE, vol. xxv. p. 52).

But I am not aware that attention has been called to the important fact, that while the individual wave rotates the wavenormal of the group remains unchanged, or, in other words, that if we fix our attention on a point moving with the group, therefore with the velocity $U$, the successive wave-planes, as they pass through that point, have all the same orientation. This follows immediately from the two formula quoted above. For the interval of time between the arrival of two successive waveplanes of similar phase at the moving point is evidently $\lambda /(V-U)$, which reduces by the first formula to $d \lambda / d V$. In this time the second of the wave-planes, having the angular velocity ad $V / d \lambda$, will rotate through an angle $a$ towards the position of the first wave-plane. But $a$ is the angle between the two planes. The second plane, therefore, in passing the moving point, will have exactly the same orientation which the first had. To get a picture of the phenomenon, we may imagine that we are able to see a few inches of the top of a moving carriagewheel. The individual spokes rotate, while the group maintains a vertical direction.

This consideration greatly simplifies the theory of Foucault's experiment, and makes it evident, I think, that the results of all such experiments depend upon the value of $U$, and not upon that of $V$.

The discussion of the experiment by following a single wave, and taking account of its rotation, is a complicated process, and one in which it is very easy to leave out of account some of the elements of the problem. The principal objection to it, however, is its unreality. If the dispersion is considerable, no wave which leaves the revolving mirror will return to it. The individual disappears, only the group has permanence. Prof. Schuster, in his communication of March II (p. 439), has nevertheless obtained by this method, as the quantity determined by " the experiments hitherto performed," $V^{2} /(2 V-U)$, which, as he observes, is nearly equal to $U$. He would, I think, have obtained $U$ precisely, if for the angle between two successive wave-planes of similar phase, instead of $2 w \lambda / V$, he had used the more exact value $2 z \lambda / U$.

By the kindness of Prof. Michelson, I am informed with respect to his recent experiments on the velocity of light in bisulphide of carbon that he would be inclined to place the maximum brilliancy of the light between the spectral lines D and $E$, but nearer to $D$. If we take the mean between $D$ and $\mathrm{E}$, we have-

$$
\frac{K}{U}=\mathbf{I} \cdot 745, \quad \frac{K(2 V-U)}{V^{2}}=\mathrm{x} \cdot 737,
$$

$K$ denoting the velocity in vacus (see Amer. Four. Ssi., vol. xxxi. p. 64). The number observed was $\mathbf{I} 76$, "with an uncertainty of two units in the second place of decimals." This agrees best with the first formula. "The same would be true if we used values nearer to the line $D$.

New Haven, Connecticut, April I

\section{J. Willard Gibbs}

\section{The Effect of Change of Temperature on the Velocity of Sound in Iron}

I VENTURE to draw attention to an error relating to the above subject, which, originating with Wertheim, still holds a place in some of our modern books on science. According to Wertheim, the velocity of sound in iron and steel is increased by rise of temperature not extending beyond $100^{\circ} \mathrm{C}$. Now in no sense whatever is this statement correct. It is true that the longitudinal elasticity of iron, as determined by the static method, will be found greater at $100^{\circ} \mathrm{C}$. than at $0^{\circ} \mathrm{C}$., provided we begin with the lower temperature first anit the wire has not, after the original annealing, been previously raised to $100^{\circ}$ C. ; but the apparent temporary increase of elasticity is really a permanent one (Phil. Trans., part i., I883, pp. 129$13 \mathrm{I}$ ), and if the wire be repeatedly heated to $100^{\circ} \mathrm{C}$. and afterwards cooled, subsequent tests will always show a less elasticity at the higher temperature than at the lower, if sufficient rest after cooling be allowed. When, however, we come to such small molecular displacements as are involved in the passage of sound through a wire, even the apparent increase of elasticity mentioned above vanishes. I have been able to prove that, when an iron or steel wire is thrown into longitudinal vibrations, so as to produce a musical note, the pitch of this note becomes lower as we raise the temperature, even when the wire is heated for the first time after it has left the maker's hands.

It seems rather strange that this error should have so long been allowed to remain uncorrected, for it has been known for many years that the pitch of a tuning-fork made of steel is lowered by small rises of temperature, and the main part of this lowering must be due to the decrease of elasticity of the steel.

King's College, Strand, April ıo

\section{Sound-producing Apparatus of the Cicadas}

Wirh regard to the above subject, treated of in an article by Mr. Lloyd Morgan in February last (NATURE, February r 8, p. 368 ), I may mention that some time ago I examined the drum of the common cicadas found plentifully in the Himalaya near Simla, and which in the evenings send forth a deafening roar from the rhododendron-trees like the whirr of large machinery. Generally the arrangement of the drum and the powerful muscles was as figured by Mr. Morgan, but I also noticed the following particulars not mentioned by him.

The chitinous rods in the membrane of the drum were not parallel, but converged slightly towards one point of the mem- 